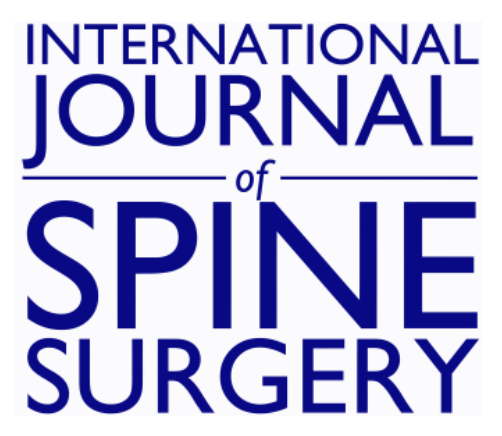

\title{
Dual-Energy Computed Tomography in Spine Fractures: A Systematic Review and Meta-Analysis
}

\author{
Henrik C. Bäcker, Chia H. Wu, Carsten Perka and Gergely Panics
}

Int J Spine Surg 2021, 15 (3) 525-535

doi: https://doi.org/10.14444/8074

http://ijssurgery.com/content/15/3/525

This information is current as of April 26, 2023.

Email Alerts Receive free email-alerts when new articles cite this article. Sign up at: http://ijssurgery.com/alerts 


\title{
Dual-Energy Computed Tomography in Spine Fractures: A Systematic Review and Meta-Analysis
}

\author{
HENRIK C. BÄCKER, MD, ${ }^{1}$ CHIA H. WU, MD, ${ }^{2}$ CARSTEN PERKA, MD, ${ }^{1}$ GERGELY PANICS, MD ${ }^{3}$ \\ ${ }^{I}$ Department of Orthopaedic Surgery and Traumatology, Charité Berlin, University Hospital, Berlin, Germany ${ }^{2}$ Department of Orthopedics \& Sports Medicine, \\ Baylor College of Medicine Medical Center, Houston, Texas, USA ${ }^{3}$ Department of Orthopaedic Surgery and Traumatology, Uszoki Hospital, Budapest, \\ Hungary
}

\begin{abstract}
Background: The purpose of this study was to perform a systematic literature review and meta-analysis to evaluate the sensitivity, specificity, and accuracy of dual-energy computed tomography (DE-CT) of bone marrow edema and disc edema in spine injuries.

In vertebral injuries, prompt diagnosis is essential to avoid any delays in treatment. Conventional radiography may only reveal indirect signs of fractures, such as when it is displaced. Therefore, to detect the presence of bone marrow or disc edemas, adjunctive tools are required, such as magnetic resonance imaging (MRI) or DE-CT.

Methods: Search terms included ((DECT) OR (DE-CT) OR (dual-energy CT) OR "Dual energy CT" OR (dualenergy computed tomography) OR (dual energy computed tomography)) AND ((spine) OR (vertebral)), and the PubMed, EMBASE, and MEDLINE databases and the Cochrane Library and Google were used. We found 1233 articles on our preliminary search, but only 13 articles met all criteria. Data were extracted to calculate the pooled sensitivity, specificity, and diagnostic odds ratio for analysis using R software.

Results: Within the 13 studies, 515 patients, 3335 vertebrae, and 926 acute fractures (27.8\%) defined by MRI were included. The largest cohort included 76 patients with 774 vertebrae. In 12 publications, MRI was reported for comparison. For DE-CT, the overall sensitivity was $86.2 \%$ with a specificity of $91.2 \%$ and accuracy of $89.3 \%$. Furthermore, 5 studies reported the accuracy of CT with an overall sensitivity of $81.3 \%$, specificity of $80.7 \%$, and accuracy with $80.9 \%$. Significant differences were found for specificity $(P<.001)$ and accuracy $(P=.023)$. However, significant interobserver differences were reported.

Conclusions: DE-CT seems to be a promising diagnostic tool to detect bone marrow and disc edemas, which can potentially replace the current gold standard, the MRI.

Level of Evidence: 2.

Clinical Relevance: This study shows that DE-CT seems to be a promising diagnostic tool with an accuracy of $89.3 \%$.
\end{abstract}

New Technology

Keywords: dual-energy, computed tomography, CT, spine, vertebra, DECT, bone marrow edema, disc edema

\section{INTRODUCTION}

Vertebral fractures can be life-threatening and cause disabilities if not diagnosed in a timely manner. Therefore, prompt diagnosis is necessary to avoid any delays in treatment. The gold standard includes conventional radiography and computed tomography (CT) where most fractures can be diagnosed. Hereby, conventional radiography may only reveal indirect signs of fractures, such as when it is displaced. Obtaining multiple views, including standing, sitting, and supine views, improves the ability to detect fractures. In the acute trauma setting, CT is typically limited to the supine position, raising concerns that injuries may be underdiagnosed or even missed. However, these tools do not allow for the assessment of the presence of bone marrow edema or disc edema, which are uncommon after fracture consolidation. As such, further studies are often required to exclude nondisplaced fractures with concomitant soft tissue injuries. Typically, the gold standard for the detection of vertebral injuries and relevant soft tissue injury is magnetic resonance imaging (MRI). ${ }^{1}$ In an acutely injured spine, MRI is better able to detect diagnoses, such as bone marrow edema or acute spinal stenosis. However, use of MRI is limited by its time-intensive nature and high costs, and patients need to be free of any 


\section{PRISMA 2009 Flow Diagram}

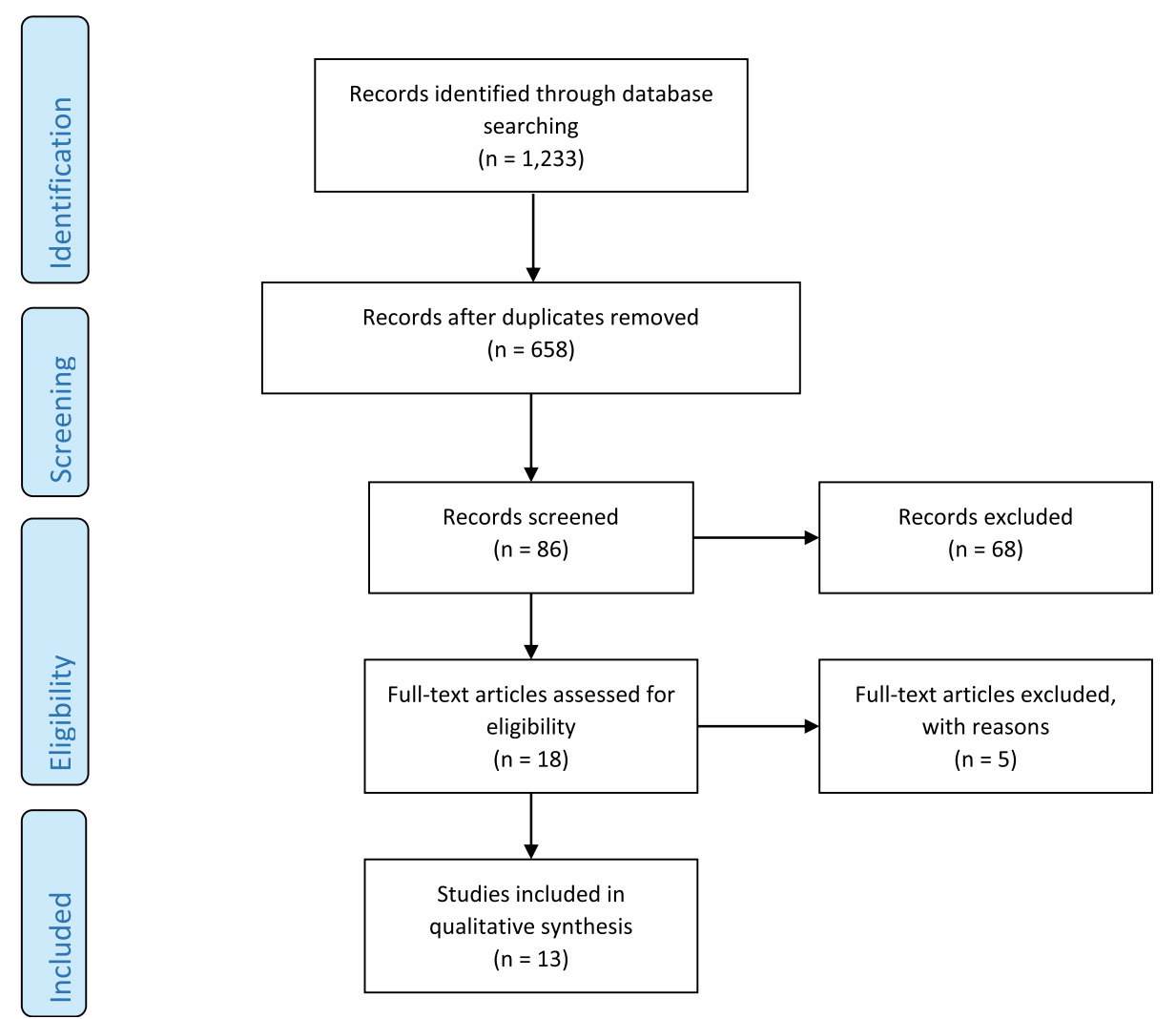

Figure 1. Included articles according to the PRISMA guidelines.

implanted metal devices, such as pacemakers or brain stimulators. ${ }^{2-4}$

Dual-energy computed tomography (DE-CT) was first described by Brooks in 1977, which has gained popularity in recent years. ${ }^{5,6}$ It measures the electron density and effective atomic number, which can be converted to Hounsfield numbers by normalizing them to water. ${ }^{7,8}$ This new tool enables visualization of bone marrow abnormalities directly, which may help detect traumatic or osteoporotic fractures. ${ }^{9}$ A variety of different indications have been described, including the spine, hip, knee, and ankle with implants as well as metabolic diseases, such as gout or neoplasm. ${ }^{10}$ Several studies have shown that DE-CT can be useful in the management of metabolic disease. ${ }^{11}$

The purpose of this study is to perform a systematic literature review and meta-analysis to evaluate the sensitivity, specificity, and accuracy of DE-CT of bone marrow edema and disc edema in spine injuries.

\section{METHODS}

\section{Systematic Review}

On December 1, 2019, a systematic review using the PRISMA guidelines was performed. ${ }^{12}$ The PubMed, EMBASE, and MEDLINE databases and the Cochrane Library and Google were used, and search terms included ((DECT) OR (DE-CT) OR (dual-energy CT) OR "Dual energy CT" OR (dual-energy computed tomography) OR (dual energy computed tomography)) AND ((spine) OR (vertebral)). All articles in French, German, and English investigating the sensitivity, specificity, and accuracy of DE-CT in spine injuries were included. Any duplicate results, lack of full access to the original article, review articles, and editorial articles were excluded. In total, 1233 articles were found on our preliminary search, but only 13 articles met all criteria. These 13 articles are included in our systematic review and meta-analysis (Figure 1). 


\section{Statistical Evaluation}

For the meta-analysis, we extracted data on the authorship, year of publication, study design (prospective or retrospective), and demographic characteristics of the participants, such as age, sample size, number of scanned vertebrae, CT, and MRI scanner. Furthermore, the true positive, false positive, true negative, and false negative results were collected and used for the metaanalysis.

The data obtained were used to calculate the pooled sensitivity and specificity as well as positive predictive value (PPV), negative predictive value (NPV), positive and negative likelihood ratios, and the diagnostic odds ratio. All sensitivities, specificities and diagnostic odds ratios were illustrated in forest plots from individual studies. To pool the sensitivity and specificity, a bivariate random effect model was applied. ${ }^{13-15}$ Further, the random effects model the method-of-moments (DerSimonianLaird) was used to calculate the $\tau^{2}$ as well as the $I^{2}$ test to assess heterogeneity. Hereby, $I^{2}$ was defined as no presence of heterogeneity between $0 \%$ and $40 \%$, moderate heterogeneity between $30 \%$ and $60 \%$, substantial heterogeneity between $50 \%$ and $90 \%$, and considerable heterogeneity between $70 \%$ and $100 \% .^{16}$ To analyze the correlation between the sensitivity and false positive rate, the Spearman's correlation coefficient was calculated, whereby a coefficient of $>.6$ was thought to be considerable.

For statistical analysis, $\mathrm{R}$ software version 4.0.3 (R Foundation for Statistical Computing, Vienna, Austria) was used, applying the mada package. All continuous variables are presented as means and a $95 \%$ confidence interval. Categorical variables were stated as percentages, and statistical significance was set to $P<.05$.

In all studies, MRI was used as a standard of reference. The accuracy per vertebra was investigated when compared with DE-CT. Furthermore, Karaca et al as well as Bierry et al distinguished not only between the imaging modalities but also between the height of vertebral injury for both thoracic and lumbar segments. Diekhoff et al compared the DE-CT findings with and without prior spine surgery. Foti et al defined a per vertebrabased $50-\mathrm{HU}$ cutoff. ${ }^{17-20}$ In a further study, different material decomposition, including adaptive iterative dose reduction with different iterations (mild, standard, and strong), were investigated. ${ }^{21}$

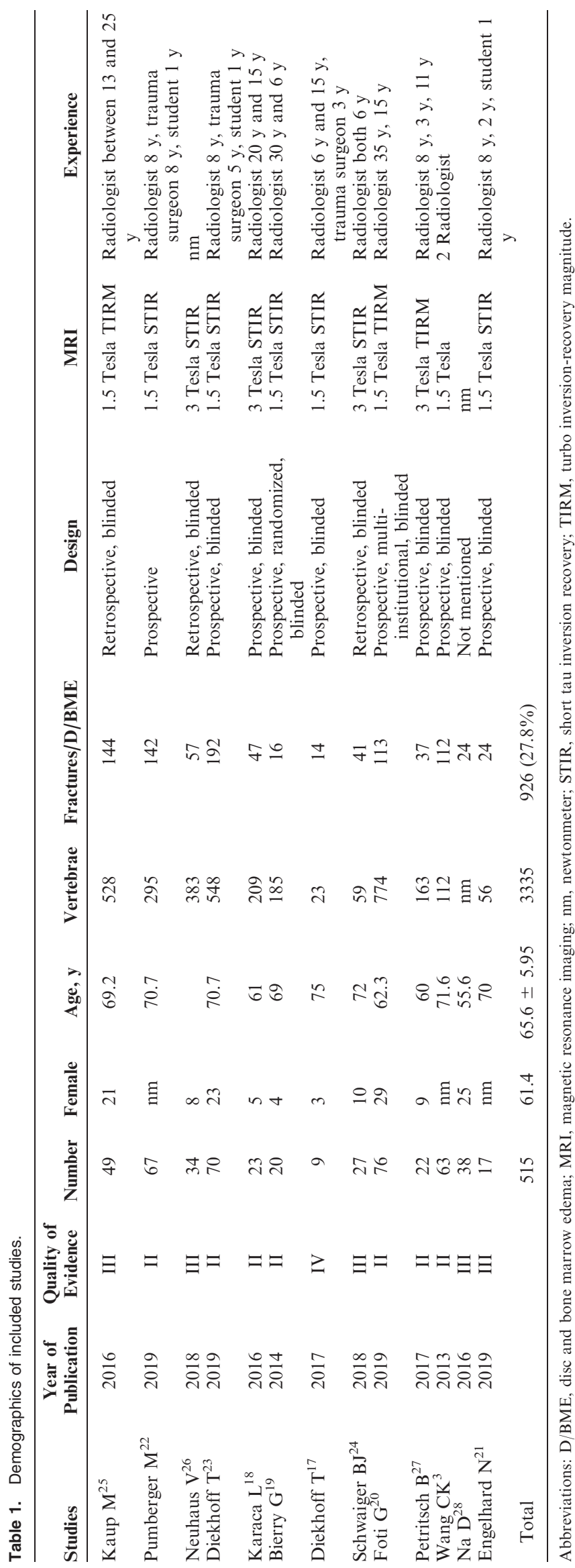


Table 2. Meta-analysis and findings for dual-energy computed tomography. ${ }^{a}$

\begin{tabular}{|c|c|c|c|c|c|c|c|c|c|c|c|}
\hline Studies & $\begin{array}{c}\text { Year of } \\
\text { Publication }\end{array}$ & Interobserver & Intraobserver & TP & $\mathbf{F P}$ & $\mathbf{F N}$ & $\mathbf{T N}$ & Sensitivity & $95 \% \mathrm{CI}$ & Specificity & $95 \% \mathrm{CI}$ \\
\hline Kaup $\mathbf{M}^{25}$ & 2016 & $\mathrm{~nm}$ & $\mathrm{~nm}$ & 56 & 6 & 6 & 46 & 90.3 & $80.1-96.4$ & 88.5 & $76.6-95.7$ \\
\hline Reader 1 & & & & 51 & 11 & 8 & 44 & 82.3 & $70.5-90.8$ & 84.6 & $71.9-93.1$ \\
\hline Reader 2 & & & & 57 & 5 & 12 & 40 & 91.9 & $82.2-97.3$ & 76.9 & $63.2-87.5$ \\
\hline Reader 3 & & & & 58 & 4 & 5 & 47 & 93.6 & $84.3-98.2$ & 90.4 & $79.0-96.8$ \\
\hline Reader 4 & & & & 57 & 5 & 5 & 47 & 91.9 & $82.2-97.3$ & 90.4 & $79.0-96.8$ \\
\hline Reader 5 & & & & 58 & 4 & 2 & 50 & 93.5 & $84.3-98.2$ & 96.2 & $86.8-99.5$ \\
\hline Pumberger $\mathrm{M}^{22}$ & 2019 & 0.51 & $\mathrm{~nm}$ & 165 & 29 & 25 & 76 & 85.1 & $79.2-89.8$ & 75.2 & $65.7-83.3$ \\
\hline Neuhaus $\mathrm{V}^{26}$ & 2018 & 0.91 & $\mathrm{~nm}$ & 45 & 5 & 1 & 304 & 97.8 & $88.5-100.0$ & 98.4 & $96.3-99.5$ \\
\hline Reader 1 & & & & 45 & 6 & 1 & 304 & 88.2 & $76.1-95.6$ & 99.7 & $98.2-100.0$ \\
\hline Reader 2 & & & & 44 & 4 & 1 & 303 & 91.7 & $80.0-97.7$ & 99.7 & $98.2-100.0$ \\
\hline Diekhoff $\mathrm{T}^{23}$ & 2019 & & & & & & & & & & \\
\hline Without prior surgery & & $0.73-0.90$ & $\mathrm{~nm}$ & 84 & 22 & 16 & 38 & 79.2 & $70.3-86.5$ & 70.4 & $56.4-82.0$ \\
\hline With prior surgery & & & & 12 & 14 & 2 & 4 & 46.2 & $26.6-66.6$ & 66.7 & $22.3-95.7$ \\
\hline Karaca $\mathrm{L}^{18}$ & 2016 & & & & & & & & & & \\
\hline Edema & & 0.82 & 0.80 & 42 & 5 & 2 & 160 & 89.4 & $76.9-96.5$ & 98.8 & $95.6-99.9$ \\
\hline Reader 1 & & & & 21 & 2 & 1 & 100 & 91.3 & $72.0-98.9$ & 99.0 & $94.6-100.0$ \\
\hline Reader 2 & & & & 21 & 3 & 1 & 60 & 87.5 & $67.6-97.3$ & 98.4 & $91.2-100.0$ \\
\hline Bierry $\mathrm{G}^{19}$ & 2014 & 0.75 & 0.86 & 21 & 5 & 4 & 155 & 80.8 & $60.7-93.5$ & 97.5 & $93.7-99.3$ \\
\hline Thoracic & & 0.76 & 0.9 & 11 & 2 & 2 & 70 & 84.6 & $54.6-98.1$ & 97.2 & $90.3-99.7$ \\
\hline Lumbar & & 0.74 & 0.81 & 10 & 3 & 2 & 85 & 76.9 & $46.2-95.0$ & 97.7 & $91.9-99.7$ \\
\hline Diekhoff $\mathrm{T}^{17}$ & 2017 & $0.63-0.89$ & $\mathrm{~nm}$ & 7 & 1 & 0 & 15 & 87.5 & 47.4-99.7 & 100.0 & $78.2-100.0$ \\
\hline Reader 1 & & & & 7 & 0 & 3 & 13 & 100.0 & $59.0-100.0$ & 81.3 & $54.4-96.0$ \\
\hline Reader 2 & & & & 7 & 1 & 0 & 15 & 87.5 & $47.4-99.7$ & 100.0 & $78.2-100.0$ \\
\hline Reader 3 & & & & 6 & 2 & 0 & 15 & 75.0 & $34.9-96.8$ & 100.0 & $78.2-100.0$ \\
\hline Schwaiger $\mathrm{BJ}^{24}$ & 2018 & 0.96 & 0.92 & 39 & 2 & 2 & 16 & 95.1 & $83.5-99.4$ & 88.9 & $65.3-98.6$ \\
\hline Reader 1 & & & & 38 & 3 & 2 & 16 & 92.7 & $80.1-98.5$ & 88.9 & $65.3-98.6$ \\
\hline Reader 2 & & & & 39 & 2 & 2 & 16 & 95.1 & $83.5-99.4$ & 88.9 & $65.3-98.6$ \\
\hline Foti $\mathrm{G}^{20}$ & 2019 & 0.87 & 0.83 & 54 & 7 & 4 & 48 & 88.5 & $77.8-95.3$ & 92.3 & $81.5-97.9$ \\
\hline Reader 1 & & & & 55 & 6 & 5 & 47 & 90.2 & $79.8-96.3$ & 90.4 & $79.0-96.8$ \\
\hline Reader 2 & & & & 56 & 5 & 5 & 47 & 91.8 & $81.6-97.2$ & 90.4 & $79.0-96.8$ \\
\hline Petritsch $\mathrm{B}^{27}$ & 2017 & 0.85 & $\mathrm{~nm}$ & 16 & 1 & 9 & 137 & 94.1 & $71.3-99.9$ & 93.8 & $88.6-97.1$ \\
\hline Wang $\mathrm{CK}^{3}$ & 2013 & $\mathrm{~nm}$ & $\mathrm{~nm}$ & 29 & 17 & 1 & 65 & 63 & $47.6-76.8$ & 98.5 & $91.8-100.0$ \\
\hline $\mathrm{Na} \mathrm{D}^{28}$ & 2016 & & & & & & & & & & \\
\hline Before $\mathrm{Ca}^{2+}$ sub & & $\mathrm{nm}$ & $\mathrm{nm}$ & 21 & 3 & 3 & 14 & 87.5 & $67.6-97.3$ & 81.2 & $54.4-96.0$ \\
\hline Engelhard $\mathrm{N}^{21}$ & 2019 & 0.52 & $\mathrm{~nm}$ & 20 & 4 & 7 & 8 & 83.3 & $62.6-95.3$ & 53.3 & $26.6-78.7$ \\
\hline 3MD, filtered back projection & & 0.54 & $\mathrm{~nm}$ & 18 & 6 & 5 & 10 & 75.0 & $53.3-90.2$ & 66.7 & $38.4-88.2$ \\
\hline $3 \mathrm{MD}$, iterative reconstruction 1 & & & & 21 & 3 & 6 & 9 & 87.5 & $67.6-97.3$ & 60.0 & $32.3-83.7$ \\
\hline $3 \mathrm{MD}$, iterative reconstruction 2 & & & & 21 & 3 & 6 & 9 & 87.5 & $67.6-97.3$ & 60.0 & $32.3-83.7$ \\
\hline $3 \mathrm{MD}$, iterative reconstruction 3 & & & & 21 & 3 & 5 & 10 & 87.5 & $67.6-97.3$ & 66.7 & $38.4-88.2$ \\
\hline 2MD, filtered back projection & & $0.62-0.72$ & $\mathrm{~nm}$ & 1 & 23 & 1 & 14 & 4.2 & $0.1-21.1$ & 93.3 & $68.1-99.8$ \\
\hline $2 \mathrm{MD}$, iterative reconstruction 1 & & $0.05-0.24$ & $\mathrm{~nm}$ & 2 & 22 & 0 & 15 & 8.3 & $1.0-27.0$ & 100.0 & $78.2-100.0$ \\
\hline $2 \mathrm{MD}$, iterative reconstruction 2 & & & & 5 & 19 & 0 & 15 & 20.8 & $7.1-42.2$ & 100.0 & $78.2-100.0$ \\
\hline 2MD, iterative reconstruction 3 & & & & 9 & 15 & 13 & 28 & 37.5 & $18.8-59.4$ & 68.3 & $51.9-81.9$ \\
\hline Total & & & & 611 & 105 & 98 & 1085 & 86.2 & $83.4-88.6$ & 91.2 & $89.4-92.7$ \\
\hline
\end{tabular}

Abbreviations: CI, confidence interval; D/BME, disc and bone marrow edema; FN, false negative; FP, false positive; MD, material decomposition; nm, newtonmeter; NPV, negative predictive value; PPV, positive predictive value; TN, true negative; TP, true positive.

${ }^{a}$ Adaptive iterative dose reduction with different iterations: 1 , mild; 2 , standard; 3 , strong.

Table 3. Individual findings for sensitivity, specificity, positive predictive value, negative predictive value, and accuracy for computed tomographies.

\begin{tabular}{|c|c|c|c|c|c|c|c|c|c|c|c|c|c|c|}
\hline Studies & Number & Male & Age, $y$ & Vertebrae & $\begin{array}{c}\text { Fractures/ } \\
\text { D/BME }\end{array}$ & $\mathbf{T P}$ & FP & FN & $\mathbf{T N}$ & Inconcl. & Sensitivity & $95 \% \mathrm{CI}$ & Specificity & $95 \% \mathrm{CI}$ \\
\hline Kaup $\mathbf{M}^{25}$ & 49 & 21 & 69.2 & 528 & 144 & 50 & 12 & 17 & 35 & 53 & 74.6 & $62.5-84.5$ & 74.5 & $59.7-86.1$ \\
\hline Reader 1 & & & & & & 49 & 13 & 31 & 21 & 34 & 79.0 & $66.8-88.3$ & 40.4 & $27.0-54.9$ \\
\hline Reader 2 & & & & & & 54 & 8 & 23 & 29 & 34 & 87.1 & $76.2-94.3$ & 55.8 & $41.3-69.5$ \\
\hline Reader 3 & & & & & & 51 & 11 & 11 & 41 & 21 & 82.3 & $70.5-90.8$ & 78.8 & $65.3-88.9$ \\
\hline Reader 4 & & & & & & 46 & 16 & 11 & 41 & 17 & 74.2 & $61.5-84.5$ & 78.8 & $65.3-88.9$ \\
\hline Reader 5 & & & & & & 49 & 13 & 9 & 44 & 15 & 79.0 & $66.8-88.3$ & 83.0 & $70.2-91.9$ \\
\hline Pumberger $\mathbf{M}^{22}$ & 67 & & 70.7 & 295 & 142 & 121 & 53 & 21 & 100 & & 69.5 & $62.1-76.3$ & 82.6 & $74.7-88.9$ \\
\hline Neuhaus $\mathrm{V}^{26}$ & 34 & 8 & & 383 & 57 & 49 & 8 & 21 & 305 & & 86.0 & $74.2-93.7$ & 93.6 & $90.3-96.0$ \\
\hline Diekhoff $T^{23}$ & 70 & 23 & 70.7 & 548 & 192 & 96 & 36 & 18 & 42 & & 72.7 & $61.3-80.1$ & 70.0 & $56.8-81.2$ \\
\hline Schwaiger $\mathrm{BJ}^{24}$ & 27 & 10 & 72 & 59 & 41 & 31 & 10 & 3 & 15 & & 91.2 & $76.3-98.1$ & 60.0 & $38.7-78.9$ \\
\hline Reader 1 & & & & & & 31 & 10 & 4 & 14 & & 75.6 & $59.7-87.6$ & 77.8 & $52.4-93.6$ \\
\hline Reader 2 & & & & & & 30 & 11 & 3 & 15 & & 73.2 & $57.1-85.8$ & 83.3 & $58.6-96.4$ \\
\hline Total & 247 & 35.1 & $67.4 \pm 5.95$ & 1813 & 576 & 347 & 119 & 80 & 497 & & 81.3 & $77.2-84.9$ & 80.7 & $77.3-83.7$ \\
\hline
\end{tabular}

Abbreviations: CI, confidence interval; D/BME, disc and bone marrow edema; FN, false negative; FP, false positive; Inconcl., inconclusive; NPV, negative predictive value; PPV, positive predictive value; TN, true negative; TP, true positive. 
Table 2. Extended.

\begin{tabular}{|c|c|c|c|c|c|c|}
\hline PPV & $95 \% \mathrm{CI}$ & NPV & $95 \% \mathrm{CI}$ & Accuracy & $95 \% \mathrm{CI}$ & Incon. \\
\hline 90.3 & $81.4-95.2$ & 88.5 & $78.1-94.3$ & 89.5 & $82.3-94.4$ & \\
\hline 86.4 & $76.9-92.4$ & 80.0 & $69.8-87.4$ & 83.3 & $75.2-89.7$ & 7 \\
\hline 82.6 & $74.2-88.7$ & 88.9 & $77.3-95.0$ & 85.1 & $77.2-91.1$ & 13 \\
\hline 92.1 & $83.4-96.4$ & 92.2 & $81.9-96.8$ & 92.1 & $85.5-96.3$ & 2 \\
\hline 91.9 & $83.2-96.3$ & 90.4 & $80.2-95.6$ & 91.2 & $84.5-95.7$ & 7 \\
\hline 96.7 & $88.2-99.1$ & 92.6 & $82.9-97.0$ & 94.7 & $88.9-98.0$ & 3 \\
\hline 86.8 & $82.4-90.3$ & 72.4 & $64.8-78.9$ & 81.7 & $76.8-85.9$ & \\
\hline 90.0 & $79.0-95.6$ & 99.7 & $97.8-100.0$ & 98.3 & $96.4-99.4$ & \\
\hline 97.8 & $86.4-99.7$ & 98.1 & $96.0-99.1$ & 98.0 & $96.0-99.2$ & \\
\hline 97.8 & $86.1-99.7$ & 98.7 & $96.7-99.5$ & 98.6 & $96.7-99.5$ & \\
\hline 84.0 & $77.5-88.9$ & 63.3 & $53.4-72.3$ & 76.3 & $68.9-82.6$ & \\
\hline 85.7 & $64.3-95.2$ & 22.2 & $12.8-35.8$ & 50.0 & $31.9-68.1$ & \\
\hline 95.5 & $84.1-98.8$ & 97.0 & $93.3-98.7$ & 96.7 & $93.2-98.6$ & \\
\hline 95.5 & $74.8-99.3$ & 98.0 & $93.0-99.5$ & 97.6 & $93.1-99.5$ & \\
\hline 95.5 & $74.9-99.3$ & 95.2 & $87.4-98.3$ & 95.3 & $88.4-98.7$ & \\
\hline 84.0 & $66.2-93.4$ & 96.9 & $93.4-98.6$ & 95.1 & $91.0-98.0$ & \\
\hline 84.6 & $57.9-95.7$ & 97.2 & $90.7-99.2$ & 95.3 & $88.4-98.7$ & \\
\hline 83.3 & $55.2-95.3$ & 96.6 & $91.3-98.7$ & 95.0 & $88.7-98.4$ & \\
\hline 100.0 & 100.0 & 93.8 & $70.6-99.0$ & 95.7 & $78.1-99.9$ & \\
\hline 70.0 & $45.7-86.6$ & 100.0 & 100.0 & 87.0 & $66.4-97.2$ & \\
\hline 100.0 & 100.0 & 93.8 & $70.6-99.0$ & 95.7 & $78.1-99.9$ & \\
\hline 100.0 & 100.0 & 88.2 & $69.3-96.1$ & 91.3 & $72.0-98.9$ & \\
\hline 95.1 & $84.1-98.6$ & 88.9 & $67.2-96.9$ & 93.2 & $83.5-98.1$ & \\
\hline 95.0 & $83.7-98.6$ & 84.2 & $63.9-94.1$ & 91.5 & $81.3-97.2$ & \\
\hline 95.1 & $84.1-98.6$ & 88.9 & $67.2-96.9$ & 93.2 & $83.5-98.1$ & \\
\hline 93.1 & $84.0-97.2$ & 87.3 & $77.3-93.3$ & 90.3 & $83.3-95.0$ & \\
\hline 91.7 & $82.6-96.2$ & 88.7 & $78.5-94.4$ & 90.3 & $83.3-95.0$ & \\
\hline 91.8 & $82.7-96.2$ & 90.4 & $80.2-95.6$ & 91.2 & $84.2-95.6$ & \\
\hline 64.0 & $48.3-77.2$ & 99.3 & $95.3-99.9$ & 93.9 & $89.0-97.0$ & \\
\hline 96.7 & $80.4-99.5$ & 79.3 & $72.4-84.8$ & 83.9 & $75.8-90.2$ & \\
\hline 87.5 & $71.4-95.2$ & 81.2 & $59.4-92.8$ & 85 & $70.2-94.3$ & \\
\hline 74.1 & $61.8-83.5$ & 66.7 & $42.1-84.6$ & 71.8 & $55.1-85.0$ & \\
\hline 78.3 & $62.9-88.4$ & 62.5 & $43.3-78.4$ & 71.8 & $55.1-85.0$ & \\
\hline 77.8 & $64.9-86.9$ & 75.0 & $49.1-90.3$ & 76.9 & $60.7-88.9$ & \\
\hline 77.8 & $64.9-86.9$ & 75.0 & $49.1-90.3$ & 76.9 & $60.7-88.9$ & \\
\hline 80.8 & $66.9-89.7$ & 76.9 & $52.2-91.1$ & 79.5 & $63.5-90.7$ & \\
\hline 50.0 & $6.3-93.7$ & 37.8 & $34.2-41.6$ & 38.5 & $23.4-55.4$ & \\
\hline 100.0 & 100.0 & 40.5 & $37.7-43.5$ & 43.6 & $27.8-60.4$ & \\
\hline 100.0 & 100.0 & 44.1 & $39.1-49.2$ & 51.3 & $34.8-67.6$ & \\
\hline 40.9 & $25.9-57.9$ & 65.1 & $56.2-73.1$ & 56.9 & $44.0-69.2$ & \\
\hline 85.3 & $82.9-87.5$ & 91.7 & $90.2-93.0$ & 89.3 & $87.8-90.7$ & \\
\hline
\end{tabular}

Table 3. Extended.

\begin{tabular}{lccccc}
\hline PPV & $\mathbf{9 5 \%} \mathbf{C I}$ & NPV & $\mathbf{9 5 \%} \mathbf{C I}$ & Accuracy & $\mathbf{9 5 \%} \mathbf{C I}$ \\
\hline 80.7 & $71.5-87.4$ & 67.3 & $56.9-76.2$ & 74.6 & $65.6-82.3$ \\
61.3 & $55.0-67.2$ & 61.8 & $47.4-74.4$ & 61.4 & $51.8-70.4$ \\
70.1 & $63.0-76.4$ & 78.4 & $64.5-87.9$ & 72.8 & $63.7-80.7$ \\
82.3 & $73.0-88.8$ & 78.8 & $68.2-86.6$ & 80.7 & $72.3-87.5$ \\
80.7 & $70.8-87.8$ & 71.9 & $62.2-80.0$ & 76.3 & $67.4-83.8$ \\
84.5 & $74.8-90.9$ & 77.2 & $67.3-84.8$ & 80.9 & $72.5-87.6$ \\
85.2 & $79.4-89.6$ & 65.4 & $59.8-70.6$ & 74.9 & $69.6-79.8$ \\
70.0 & $60.4-78.1$ & 97.4 & $95.3-98.6$ & 92.4 & $89.3-94.9$ \\
84.2 & $78.1-88.8$ & 53.8 & $45.8-61.7$ & 71.9 & $65.0-78.1$ \\
75.6 & $65.5-83.5$ & 83.3 & $61.8-93.9$ & 78.0 & $65.3-87.7$ \\
88.6 & $76.2-94.9$ & 58.3 & $43.6-71.7$ & 76.3 & $63.4-86.4$ \\
90.9 & $77.8-96.6$ & 57.7 & $44.1-70.2$ & 76.3 & $63.4-86.4$ \\
74.5 & $71.2-77.5$ & 86.1 & $83.6-88.4$ & 80.9 & $78.4-83.3$ \\
\hline
\end{tabular}

Seven of 13 articles included report the accuracy of conventional CT.

\section{RESULTS}

In total, 515 patients, 3335 vertebrae, and 926 fractures $(27.8 \%)$ were included. Three studies were retrospective, and 9 authors reported that they blinded their cohort. Most of the studies assessed individual vertebrae. ${ }^{17,22,23}$ In one study, the primary end point only included bone edema, ${ }^{18}$ and 6 studies performed additional comparisons with $\mathrm{CT}$. One publication described a different cutoff for the Hounsfield unit of $50 \mathrm{HU}$, whereas another study described different reconstruction algorithms, such as material decomposition and calcium subtraction..$^{20,21}$ Likewise, the number of DE-CT image interpreters varied as well across studies, ranging between 2 readers ${ }^{18,20,24}$ and 5 readers. ${ }^{25}$ Furthermore, different MRI sequences were used in different studies. The majority of authors used a 1.5-Tesla ${ }^{3,17,19-23,25}$ and short tau inversion recovery sequence. ${ }^{17,18,19,21-24,26}$ Remaining studies used turbo inversion-recovery magnitude sequences. ${ }^{20,25,27}$ One study did not report MR tomography for comparison. ${ }^{28}$

The mean age of patients was $67.6 \pm 5.95$ years old. Females represented $61.4 \%(n=205 / 334)$ of cases. In 2 studies, no sex distribution was reported (Table 1). ${ }^{22,26}$ Two studies did not describe detailed individual DE-CT findings, only the overall sensitivity, specificity, and accuracy. ${ }^{22,27}$

The largest cohort included 76 patients with 774 vertebrae. $^{20,23}$ Among the individual DE-CT readers, the sensitivity for two-material decomposition filtered back projection ranged from $4.2 \%,{ }^{21}$ $76.9 \%{ }^{19}$ to $100 \%{ }^{17,24}$ and specificity per vertebrae for two-material decomposition raw data ranged from $53.3 \%,{ }^{21} 66.7 \%,{ }^{23}$ to $100 \% .{ }^{17}$ The overall sensitivity was $86.2 \%$ with a specificity of $91.2 \%$. PPV was $85.3 \%$ and NPV was $91.7 \%$. The accuracy for two-material decomposition filtered back projection ranged from $38.5 \%,{ }^{21} 50 \%,{ }^{17}$ to $98.6 \%,{ }^{26}$ with a mean of $89.3 \%$. All findings for DE-CT are presented in Table 2. Heterogeneity was found to be considerable (Higgins $I^{2}>70 \%$ ) in all cases (sensitivity, specificity, and diagnostic odds ratio). All findings are illustrated in Figures 2 and 3.

For CT readings, the sensitivity ranged from $69.5 \%{ }^{22}$ to $87.1 \%{ }^{25}$ and specificity was between $40.4 \%{ }^{25}$ and $93.6 \%{ }^{26}$ Average sensitivity was reported to be $81.3 \%$ and $80.7 \%$ for specificity. The mean PPV was $74.5 \%$ and NPV was $86.1 \%$, 
a Study

Kaup M

Pumberger $\mathrm{M}$

Neuhaus $V$

Diekhoff T 2019 - Without prior surgery

Diekhoff T 2019 - With prior surgery

Karaca $\mathbf{L}$

Bierry G - Thoracic

Bierry G - Lumbar

Diekhoff T 2017

Schwaiger BJ

Foti G

Petritsch B

Wang CK

$\mathrm{Na} \mathrm{D}$

Engelhard $\mathrm{N}$

Random effects mode

Heterogeneily: $l^{2}=72 \%, \tau^{2}=0.5300, p<0.01$
Events Total

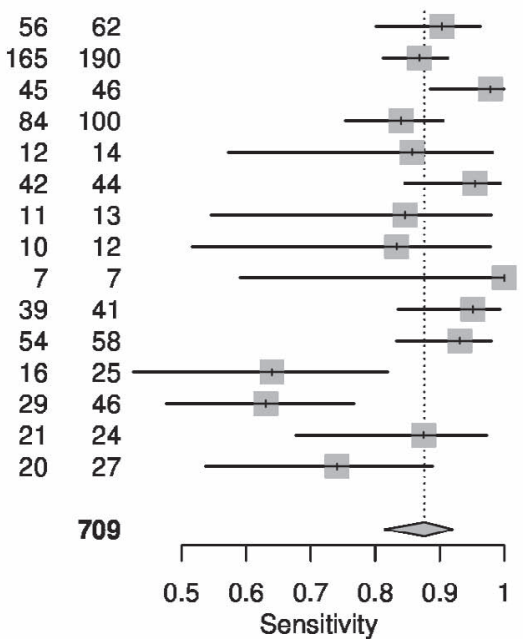

Proportion

95\%-Cl

$0.903[0.801 ; 0.964]$

0.868 [0.812; 0.913$]$

$0.978[0.885 ; 0.999]$

0.840 [0.753; 0.906 ]

0.857 [0.572; 0.982$]$

$0.955[0.845 ; 0.994]$

$0.846[0.546 ; 0.981]$

$0.833[0.516 ; 0.979]$

$1.000[0.590 ; 1.000]$

$0.951 \quad[0.835 ; 0.994]$

$0.931 \quad[0.833 ; 0.981]$

$0.640[0.425 ; 0.820]$

$0.630[0.475 ; 0.768]$

$0.875[0.676 ; 0.973]$

$0.741[0.537 ; 0.889]$

$0.876[0.815 ; 0.919]$

b Study

Events Total

$46 \quad 52$

Kaup M

Pumberger $\mathrm{M}$

Neuhaus $V$

Diekhoff T 2019 - Without prior surgery

Diekhoff T 2019 - With prior surgery

Karaca L

Bierry G - Thoracic

Bierry G - Lumbar

Diekhoff T 2017

Schwaiger BJ

Foti G

Petritsch B

Wang CK

$\mathrm{Na} \mathrm{D}$

Engelhard N

Random effects mode

Heterogeneily: $I^{2}=90 \%, \tau^{2}=1.9339, p<0.01$

$76 \quad 105$

$304 \quad 309$

$38 \quad 60$

$\begin{array}{rr}4 & 18 \\ 160 & 165\end{array}$

$\begin{array}{ll}70 & 72\end{array}$

$85 \quad 88$

$15 \quad 16$

$16 \quad 18$

$48 \quad 55$

$137 \quad 138$

$\begin{array}{ll}65 & 66\end{array}$

$13 \quad 16$

$8 \begin{array}{rr}13 & 16 \\ 8 & 12\end{array}$

1190

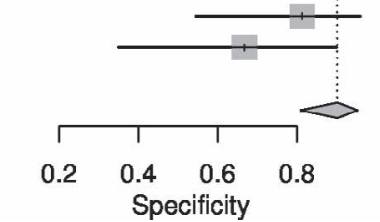

$0.885[0.766 ; 0.956]$

0.724 [0.628; 0.807$]$

$0.984[0.963 ; 0.995]$

$0.633[0.499 ; 0.754]$

$0.222[0.064 ; 0.476]$

$0.970[0.931 ; 0.990]$

$0.972[0.903 ; 0.997]$

$0.966[0.904 ; 0.993]$

$0.938[0.698 ; 0.998]$

$0.889[0.653 ; 0.986]$

$0.873[0.755 ; 0.947$

$0.993[0.960 ; 1.000]$

$0.985[0.918 ; 1.000]$

$0.812[0.544 ; 0.960]$

$0.667[0.349 ; 0.901]$

$0.902[0.809 ; 0.952]$
C study

Kaup M

Pumberger $M$

Neuhaus V

Diekhoff T 2019 - Without prior surgery

Diekhoff T 2019 - With prior surgery

Karaca L

Bierry G - Thoracic

Bierry G - Lumbar

Diekhoff T 2017

Schwaiger BJ

Foti $\mathrm{G}$

Petritsch B

Wang CK

$\mathrm{Na} D$

Engelhard N

Random effects mode

Heterogeneity: $l^{2}=82 \%, \tau^{2}=2.1939, p<0.01$

\begin{tabular}{rrrr}
$\begin{array}{c}\text { Experimental } \\
\text { Events }\end{array}$ Total Events & \multicolumn{2}{c}{ Control } \\
Total \\
56 & 62 & 6 & 52 \\
165 & 194 & 25 & 101 \\
45 & 50 & 1 & 305 \\
84 & 106 & 16 & 54 \\
12 & 26 & 2 & 6 \\
42 & 47 & 2 & 162 \\
11 & 13 & 2 & 72 \\
10 & 13 & 2 & 87 \\
7 & 8 & 0 & 15 \\
39 & 41 & 2 & 18 \\
54 & 61 & 4 & 52 \\
16 & 17 & 9 & 146 \\
29 & 30 & 17 & 82 \\
21 & 24 & 3 & 16 \\
20 & 24 & 7 & 15 \\
& & & \\
& 716 & & 1183
\end{tabular}

Odds Ratio

OR

$95 \%-\mathrm{Cl}$

71.556 [21.619; 236.840] 17.297 [ 9.493; 31.515] 736.000 [312.473; 23956.331] $9.068 \quad$ [ 4.286; 19.185] $1.714 \quad[0.266 ; 11.060]$ 672.000 [125.914; 3586.459] 192.500 [24.523; 1511.068]

141.667 [ $21.075 ; 952.298]$

155.000 [ $5.619 ; 4275.434]$

156.000 [ 20.193; 1205.166]

92.571 [25.520; 335.799]

243.556 [28.947; 2049.256]

110.882 [14.080; 873.233]

30.333 [ $5.306 ; 173.406]$

5.714 [ $1.305 ; 25.026$ ]

63.771 [ 26.572; 153.048]

Figure 2. (a) Forest plots for sensitivity, (b) specificity, and (c) diagnostic odds ratio showing the individual proportions including $95 \%$ confidence interval and the heterogeneity. 
SROC curve (bivariate model) for Diagnostic Test Accuracy

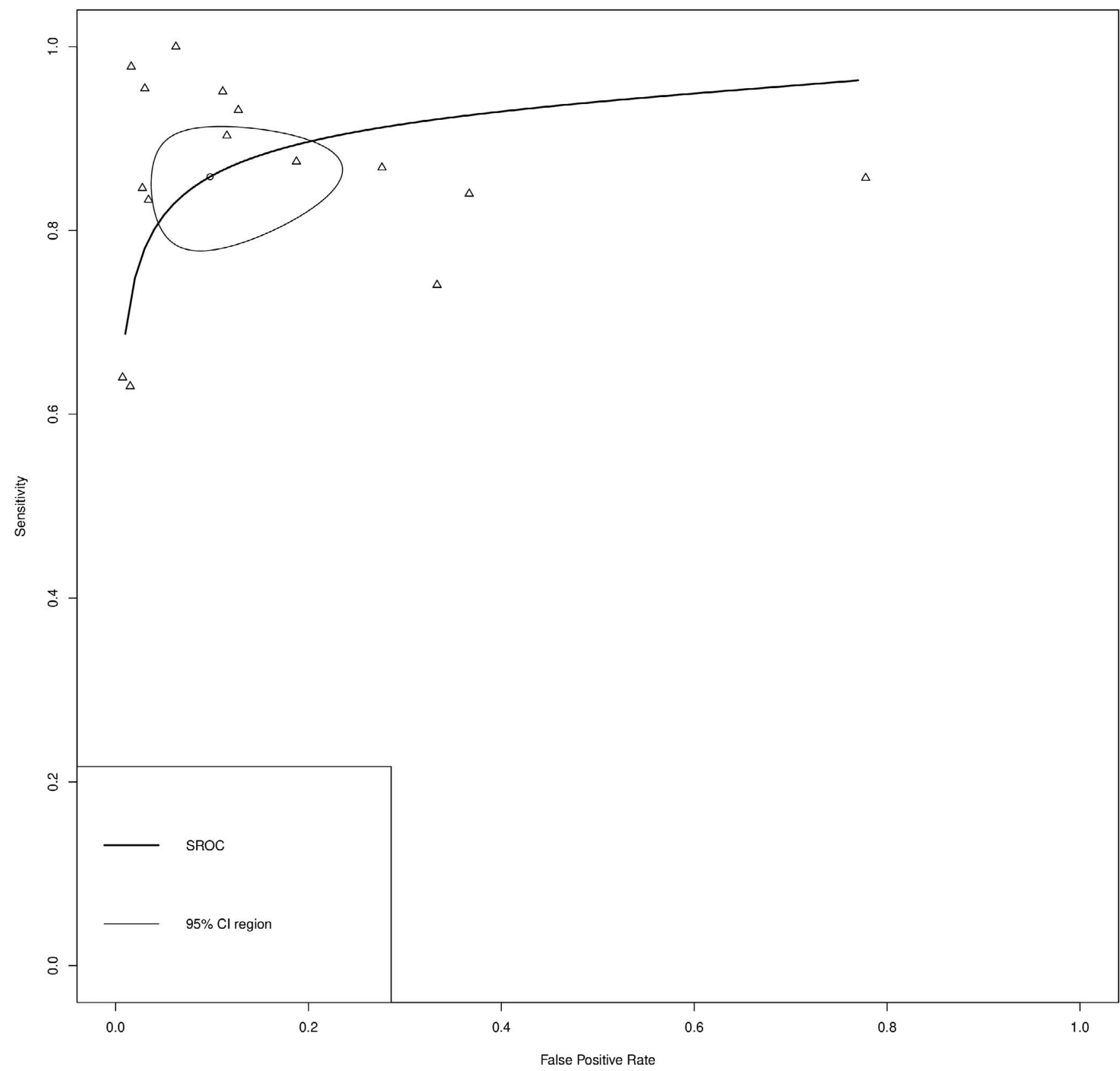

Figure 3. SROC (summary receiver operating characteristic) curve for diagnostic test accuracy between sensitivity and false positive rate for dual-energy computed tomography.

with a mean accuracy of $80.9 \%$ (Table 3 ). Hereby, the sensitivity showed only moderate heterogeneity (Higgins $I^{2}=52 \%$ ), whereas for the specificity and diagnostic odds ratio, this was considerable (Figures 4 and 5).

Specificity and accuracy reported above were found to be statistically significant at $P<.001, P=$ .067 , and $P=.029$, respectively. No significance was found for sensitivity at a $P$ value of .119 .

\section{DISCUSSION}

This systematic review included 13 studies, totaling 515 patients, 3335 vertebrae, and 926 fractures. Although DE-CT shows higher sensitivity, specificity, and accuracy when compared with conventional CT, significant interobserver differences were identified. Previous literature reviews on DE-CT did not focus on its use in the context of 

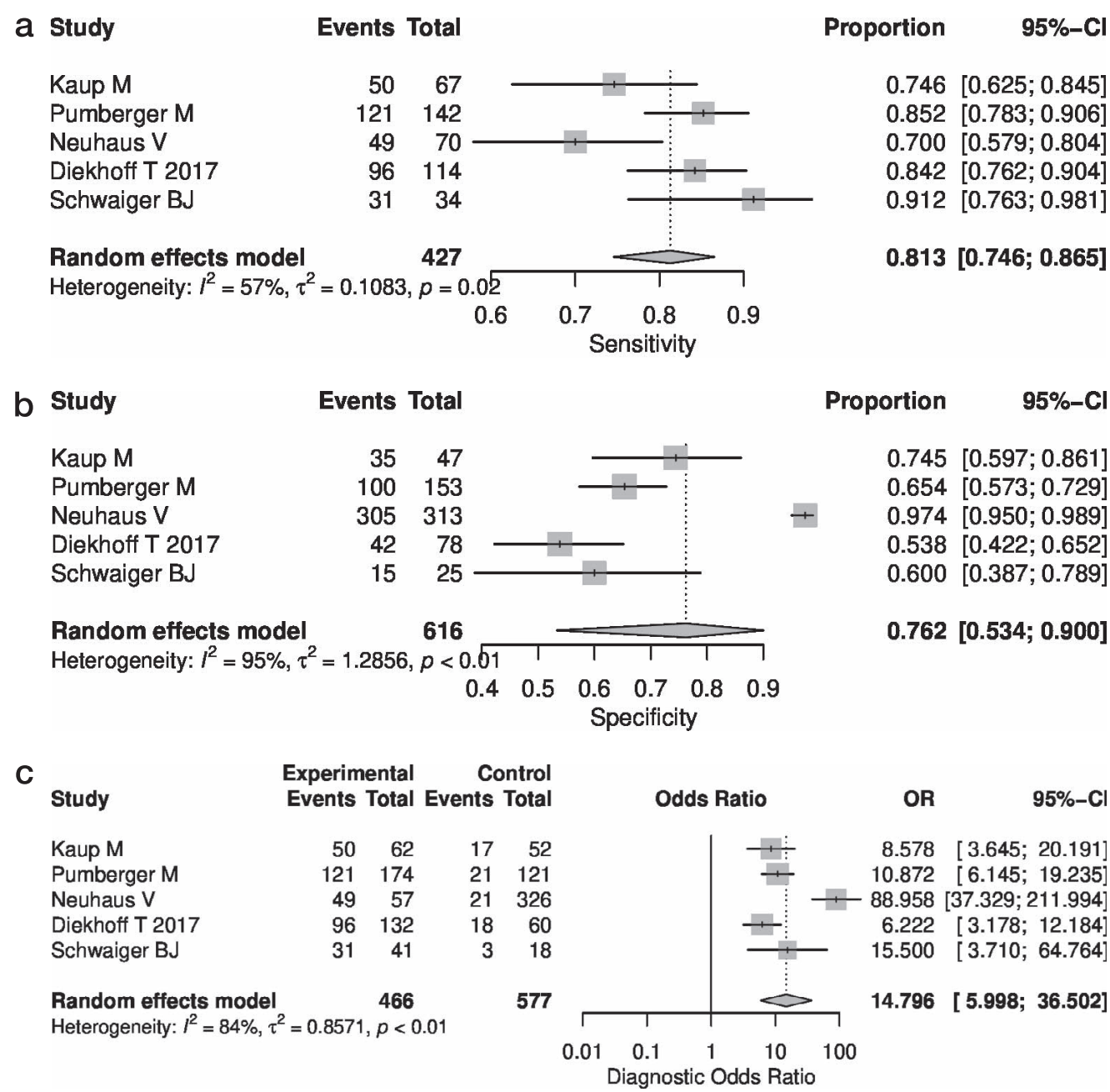

Figure 4. (a) Forest plots for sensitivity, (b) specificity, and (c) diagnostic odds ratio showing the individual proportions including $95 \%$ confidence interval and the heterogeneity.

spine injury. ${ }^{10,29,30}$ Our review is the first study of its kind to our knowledge.

For conventional CT scans, the sensitivity was between $69.5 \%$ and $87.1 \%$, and specificity was between $40.4 \%$ and $93.6 \%$, with a PPV ranging from $61.3 \%$ to $90.9 \%$ and an NPV ranging from $53.8 \%$ to $97.4 \%$. For DE-CT, the sensitivity ranged from $76.9 \%$ to $100 \%$ and specificity from $66.7 \%$ to $100 \%$. PPV was found to be between $64 \%$ and $100 \%$ and NPV between $22.2 \%$ and $100 \%$. However, Engelhard et al reported high variability for different reconstruction algorithms of DE-CT; twomaterial decomposition with filtered back projection showed the lowest sensitivity of $4.2 \%$ and specificity for raw data at $53.3 \% .^{21}$

Some differences were observed between the individual reader sensitivity and specificities. According to Pumberger et al, the sensitivity was much higher for a radiologist at $89 \%$, a medical student at $86 \%$, and a specialized orthopaedic surgeon at $73 \%$.
For specificity, radiologists were reported at $93 \%$, orthopaedic surgeons at $59 \%$, and medical students at $54 \% .^{22}$

According to Bierry et al, accuracy of DE-CT was superior for the thoracic spine as compared with the lumbar spine. While a 50-HU cutoff has been reported to be superior to attenuation measurement of bone bruises in the knee joint, the investigation by Foti et al regarding the usefulness of the 50-HU cutoff for distinguishing between fresh and old vertebral fractures did not find any statistical significance. $^{31}$

Reconstruction algorithms after calcium subtraction showed slightly better sensitivity of $91.7 \%$ when compared with reconstruction algorithms without calcium subtraction $(87.5 \%){ }^{28}$ Additionally, the three-material decomposition algorithm was found to be better than the two-material decomposition algorithm. ${ }^{32}$ For adaptive iterative dose reduction, no differences between the mild, stan- 


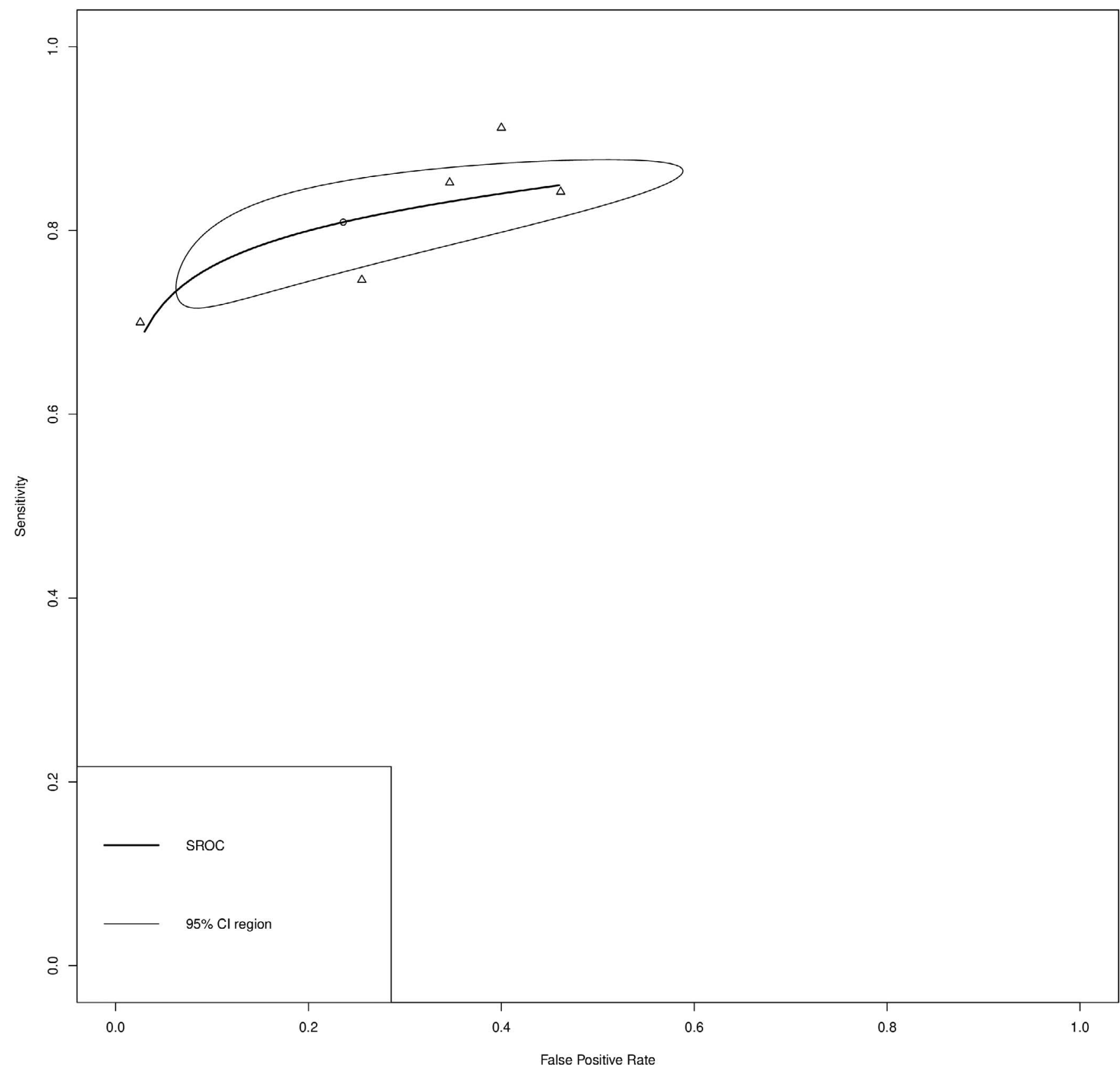

Figure 5. SROC (summary receiver operating characteristic) curve for diagnostic test accuracy between sensitivity and false positive rate for computed tomography.

dard, or moderate iterations were reported. ${ }^{21}$ In other studies, multienergy CT was observed to have better noise reduction with prior information included. $^{32}$

Besides the initial diagnostic with conventional radiography and $\mathrm{CT}$, the gold standard for diagnosing bone marrow edema and disc edema, such as in nondisplaced spine trauma, is MRI, although its ability to detect fractures may vary depending on the etiology, namely osteoporotic versus malignant causes. ${ }^{1,33}$ MRI identifies subtle bony edema seen in compression fractures that would otherwise be missed by conventional radiography. For stress fractures in the lumbar spine, the sensitivity was reported at $99.6 \%$, specificity at $86.7 \%$, and accuracy at $97.2 \%$. Higher accuracy has been reported at the lower lumbar levels. ${ }^{34}$ Interestingly, greater interobserver variation was observed in patients with intact pars interarticularis. $^{35}$ 
According to Lenchik et al, DE-CT sensitivity for bone marrow edema in osteoporotic vertebral fractures is described to be $92 \%$ with a specificity of $96 \%$. Yang et al performed a meta-analysis on 7 studies assessing the accuracy of DE-CT for vertebral fractures. Sensitivity and specificity was described to be $89 \%$ and $98 \%$, respectively. ${ }^{30}$ This is much higher than what was found in our systematic review. ${ }^{36}$ Although CT shows less accuracy than DE-CT for detecting vertebral fractures, the experience and familiarity of most image interpreters with regular CT may bias this result. Another important factor is the time elapsed between the accident and the time of imaging, as the sensitivity increased if the interval was more than 2 days. ${ }^{30}$ Use of MRI is limited by metal implants in patients, availability of MRI in clinics, and individual risk factors, such as cardiopulmonary instability or claustrophobia. In those cases, DE-CT can be a reasonable alternative to MRI.

A DE-CT, uses 2 scintillation layers (2 sources of $\mathrm{x}$-ray), 1 normal and a second less powerful x-ray with 2 corresponding detectors. In rapid $\mathrm{kVp}$ switching, the tube voltages follow a pulsed curve, which is collected twice for every projection at high and low tube voltage. Therefore, the 2 scintillation layers enable a separation between the high and low energy spectra. ${ }^{37}$

There are several limitations to this study as this is a systematic analysis and meta-analysis. Four studies were performed by Dr Diekhoff's group. ${ }^{17,21-23}$ Individual findings for the different observers were not consistently reported. Only a few studies reported both the intraobserver and interobserver reliability. ${ }^{18-20,24}$ Furthermore, different kinds of MRI were used as gold standards for comparison to detect bone marrow and disc edemas. Although all studies wanted to report the accuracy of DE-CT, the studies did not use the same reconstruction algorithms or cutoffs across the board, making comparison difficult. The largest cohort included only 76 patients. ${ }^{20,23}$

\section{CONCLUSION}

DE-CT seems to be a promising diagnostic tool to exclude bone marrow and disc edema in any acute spine injuries where MRI cannot be performed, as evidenced by our systematic review and meta-analysis. However, the literature still supports the use of MRI as the gold standard. But as familiarity and access to DE-CT improves, inter- and intraobserver agreement will likely improve as well. DE-CT has the potential to replace MRI as the diagnostic modality of choice for spine injuries in some settings.

\section{REFERENCES}

1. Atsina KB, Rozenberg A, Selvarajan SK. The utility of whole spine survey MRI in blunt trauma patients sustaining single level or contiguous spinal fractures. Emerg Radiol. 2019;26(5):493-500.

2. Komlosi P, Wintermark M. Dual energy computed tomography applications for the evaluation of the spine. Neuroimaging Clin N Am. 2017;27(3):483-487.

3. Wang CK, Tsai JM, Chuang MT, Wang MT, Huang KY, Lin RM. Bone marrow edema in vertebral compression fractures: detection with dual-energy CT. Radiology. 2013;269(2):525-533.

4. Tanigawa N, Komemushi A, Kariya S, et al. Percutaneous vertebroplasty: relationship between vertebral body bone marrow edema pattern on MR images and initial clinical response. Radiology. 2006;239(1):195-200.

5. Brooks RA. A quantitative theory of the Hounsfield unit and its application to dual energy scanning. J Comput Assist Tomogr. 1977;1(4):487-493.

6. Brooks RA, Di Chiro G. Split-detector computed tomography: a preliminary report. Radiology. 1978;126(1):255-257.

7. Logan JA, Morrison E, McGill PE. Serum uric acid in acute gout. Ann Rheum Dis. 1997;56(11):696-697.

8. Dunscombe PB, Katz DE, Stacey AJ. Some practical aspects of dual-energy CT scanning. $\mathrm{Br} J$ Radiol. 1984;57(673):82-87. doi:10.1259/0007-1285-57-673-82

9. Johnson TR, Krauss B, Sedlmair M, et al. Material differentiation by dual energy CT: initial experience. Eur Radiol. 2007;17(6):1510-1517.

10. Mallinson PI, Coupal TM, McLaughlin PD, Nicolaou S, Munk PL, Ouellette HA. Dual-energy CT for the musculoskeletal system. Radiology. 2016;281(3):690-707.

11. Rech HJ, Cavallaro A. Dual-energy computed tomography diagnostics for gout. $Z$ Rheumatol. 2017;76(7):580-588.

12. Liberati A, Altman DG, Tetzlaff J, et al. The PRISMA statement for reporting systematic reviews and meta-analyses of studies that evaluate health care interventions: explanation and elaboration. J Clin Epidemiol. 2009;62(10):e1-34.

13. Kim KW, Lee J, Choi SH, Huh J, Park SH. Systematic review and meta-analysis of studies evaluating diagnostic test accuracy: a practical review for clinical researchers-part I. General Guidance and Tips. Korean J Radiol. 2015;16(6):11751187.

14. Lee J, Kim KW, Choi SH, Huh J, Park SH. Systematic review and meta-analysis of studies evaluating diagnostic test accuracy: a practical review for clinical researchers-part II. Statistical methods of meta-analysis. Korean $J$ Radiol. 2015;16(6):1188-1196.

15. Suh $\mathrm{CH}$, Park SH. Successful publication of systematic review and meta-analysis of studies evaluating diagnostic test accuracy. Korean J Radiol. 2016;17(1):5-6.

16. Higgins JP, Altman DG, Gotzsche PC, et al. The Cochrane Collaboration's tool for assessing risk of bias in 
randomised trials. $B M J$. 2011;343:d5928. doi:10.1136/bmj. d5928

17. Diekhoff T, Hermann KG, Pumberger M, Hamm B, Putzier M, Fuchs M. Dual-energy CT virtual non-calcium technique for detection of bone marrow edema in patients with vertebral fractures: a prospective feasibility study on a singlesource volume CT scanner. Eur J Radiol. 2017;87:59-65. doi:10. 1016/j.ejrad.2016.12.008

18. Karaca L, Yuceler Z, Kantarci M, et al. The feasibility of dual-energy CT in differentiation of vertebral compression fractures. Br J Radiol. 2016;89(1057):20150300. doi:10.1259/bjr. 20150300

19. Bierry G, Venkatasamy A, Kremer S, Dosch JC, Dietemann JL. Dual-energy CT in vertebral compression fractures: performance of visual and quantitative analysis for bone marrow edema demonstration with comparison to MRI. Skeletal Radiol. 2014;43(4):485-492.

20. Foti G, Beltramello A, Catania M, Rigotti S, Serra G, Carbognin G. Diagnostic accuracy of dual-energy CT and virtual non-calcium techniques to evaluate bone marrow edema in vertebral compression fractures. Radiol Med. 2019;124(6):487-494.

21. Engelhard N, Hermann KG, Greese J, et al. Singlesource dual-energy computed tomography for the detection of bone marrow lesions: impact of iterative reconstruction and algorithms. Skeletal Radiol. 2020;49(5):765-772. doi:10.1007/ s00256-019-03330-w

22. Pumberger M, Fuchs M, Engelhard N, et al. Disk injury in patients with vertebral fractures - a prospective diagnostic accuracy study using dual-energy computed tomography. Eur Radiol. 2019;29(8):4495-4502.

23. Diekhoff T, Engelhard N, Fuchs M, et al. Single-source dual-energy computed tomography for the assessment of bone marrow oedema in vertebral compression fractures: a prospective diagnostic accuracy study. Eur Radiol. 2019;29(1):31-39.

24. Schwaiger BJ, Gersing AS, Hammel J, et al. Threematerial decomposition with dual-layer spectral CT compared to MRI for the detection of bone marrow edema in patients with acute vertebral fractures. Skeletal Radiol. 2018;47(11):1533-1540.

25. Kaup M, Wichmann JL, Scholtz JE, et al. Dual-energy CT-based display of bone marrow edema in osteoporotic vertebral compression fractures: impact on diagnostic accuracy of radiologists with varying levels of experience in correlation to MR imaging. Radiology. 2016;280(2):510-519.

26. Neuhaus V, Lennartz S, Abdullayev N, et al. Bone marrow edema in traumatic vertebral compression fractures: diagnostic accuracy of dual-layer detector CT using calcium suppressed images. Eur J Radiol. 2018;105:216-220. doi:10. 1016/j.ejrad.2018.06.009

27. Petritsch B, Kosmala A, Weng AM, et al. Vertebral compression fractures: third-generation dual-energy CT for detection of bone marrow edema at visual and quantitative analyses. Radiology. 2017;284(1):161-168.

28. Na D, Hong SJ, Yoon MA, et al. Spinal bone bruise: can computed tomography (CT) enable accurate diagnosis? Acad Radiol. 2016;23(11):1376-1383.

29. Li M, Qu Y, Song B. Meta-analysis of dual-energy computed tomography virtual non-calcium imaging to detect bone marrow edema. Eur J Radiol. 2017;95:124-129. doi:10. 1016/j.ejrad.2017.08.005

30. Yang P, Wu G, Chang X. Diagnostic accuracy of dualenergy computed tomography in bone marrow edema with vertebral compression fractures: a meta-analysis. Eur J Radiol. 2018;99:124-129. doi:10.1016/j.ejrad.2017.12.018

31. Pache G, Bulla S, Baumann T, et al. Dose reduction does not affect detection of bone marrow lesions with dual-energy CT virtual noncalcium technique. Acad Radiol. 2012;19(12):1539-1545.

32. Ren L, McCollough $\mathrm{CH}, \mathrm{Yu}$ L. Three-material decomposition in multi-energy CT: impact of prior information on noise and bias. Proc SPIE Int Soc Opt Eng. 2018;10573:105731G. doi:10.1117/12.2294953

33. Abdel-Wanis ME, Solyman MT, Hasan NM. Sensitivity, specificity and accuracy of magnetic resonance imaging for differentiating vertebral compression fractures caused by malignancy, osteoporosis, and infections. J Orthop Surg (Hong Kong ). 2011;19(2):145-150.

34. Ganiyusufoglu AK, Onat L, Karatoprak O, Enercan M, Hamzaoglu A. Diagnostic accuracy of magnetic resonance imaging versus computed tomography in stress fractures of the lumbar spine. Clin Radiol. 2010;65(11):902-907.

35. Campbell RS, Grainger AJ. Optimization of MRI pulse sequences to visualize the normal pars interarticularis. Clin Radiol. 1999;54(1):63-68.

36. Lenchik L, Rogers LF, Delmas PD, Genant HK. Diagnosis of osteoporotic vertebral fractures: importance of recognition and description by radiologists. AJR Am J Roentgenol. 2004;183(4):949-958.

37. Lam S, Gupta R, Kelly H, Curtin HD, Forghani R. Multiparametric evaluation of head and neck squamous cell carcinoma using a single-source dual-energy CT with fast $\mathrm{kVp}$ switching: state of the art. Cancers (Basel). 2015;7(4):22012216.

Disclosures and COI: The authors declare no conflict of interest related to this study.

Corresponding Author: Henrik C. Bäcker, MD, University Hospital Berlin, Charitéplatz 1, 10117 Berlin, Germany. Phone: 0049-31/450 615179; Email: henrik.baecker@sports-med.org.

Published 1 June 2021

This manuscript is generously published free of charge by ISASS, the International Society for the Advancement of Spine Surgery. Copyright (C) 2021 ISASS. To see more or order reprints or permissions, see http://ijssurgery.com. 